PROCEEDINGS OF THE

AMERICAN MATHEMATICAL SOCIETY

Volume 137, Number 10, October 2009, Pages 3477-3486

S 0002-9939(09)09991-2

Article electronically published on June 3, 2009

\title{
RANDOM $p$-ADIC RIESZ PRODUCTS: CONTINUITY, SINGULARITY, AND DIMENSION
}

\author{
NARN-RUEIH SHIEH AND XIONG-YING ZHANG
}

(Communicated by Richard C. Bradley)

\begin{abstract}
We study precise conditions for mutual absolute continuity and mutual singularity of two random $p$-adic Riesz products, defined respectively by two sequences of coefficients $a_{k}, b_{k}$. Our conditions and assertions are specific to the $p$-adic case. We also calculate explicitly the Hausdorff dimension, and in case the defining coefficients are constant, we have an integral representation of the dimension formula with a rapid convergence rate $p^{-k}$.
\end{abstract}

\section{Introduction AND MAIN RESUlts}

Let $p \geq 2$ be a prime number and let $\mathbb{Q}_{p}$ be the field of $p$-adic numbers (see [10, 14, 15, 16, for more information about $p$-adic numbers and $p$-adic analysis). The absolute value on $\mathbb{Q}_{p}$, denoted by $|\cdot|_{p}$, is non-Archimedean. The unit ball $\mathbb{Z}_{p}=\left\{x \in \mathbb{Q}_{p}:|x|_{p} \leq 1\right\}$ is a local ring, called the ring of $p$-adic integers. The $\mathbb{Q}_{p}$ and $\mathbb{Z}_{p}$ are very important non-Archimedean structures in mathematics and mathematical physics. The purpose of this paper is to study some fundamental properties of random Riesz products defined on the additive group $\left(\mathbb{Z}_{p},+\right.$ ) (in the following context, the + denotes both the real addition and the $p$-adic addition for notational convenience). Let $(\Omega, \mathscr{A}, \mathbf{P})$ be a probability space, and $\omega_{k}, k \geq 1$, be a sequence of i.i.d. random variables defined on $\Omega$, with the normalized Haar measure, denoted by $d x$, on $\mathbb{Z}_{p}$ as common probability law. The random p-adic Riesz product (measure) on $\mathbb{Z}_{p}$ denoted by $\mu_{a, \omega}$ (here, $\omega \in \Omega$ indicates the randomness) is a random measure formally expressed as

$$
d \mu_{a, \omega}=\prod_{k=1}^{\infty}\left(1+\boldsymbol{R} \mathbf{e} a_{k} \gamma_{k}\left(x+\omega_{k}\right)\right) d x,
$$

where $a:=\left\{a_{k}\right\}$ is a sequence of complex numbers with $\left|a_{k}\right| \leq 1$ and $\gamma_{k}$ is a sequence of characters of $\mathbb{Z}_{p}$ taken to be $\gamma_{k}(t)=\exp \left(2 \pi i\left\{p^{-k} t\right\}\right)$. See Section 2 for the definitions.

In this work, we will study precise conditions for the mutual absolute continuity and the mutual singularity of such two Riesz products $\mu_{a, \omega}, \mu_{b, \omega}$ on $\mathbb{Z}_{p}$, and also calculate explicitly the Hausdorff dimension of such $\mu_{a, \omega}$. The study of mutual absolute continuity and mutual singularity of infinite product measures has a long

Received by the editors June 9, 2008.

2000 Mathematics Subject Classification. Primary 60G57, 28A80, 11S80.

Key words and phrases. Random $p$-adic Riesz products, mutual absolute continuity, mutual singularity, Hausdorff dimension.

(C)2009 American Mathematical Society Reverts to public domain 28 years from publication 
history since the seminal work of Kakutani ([8]). We mention that such a study for both deterministic and random Riesz products on the circle has been investigated intensively by various authors $([1,0,12,13])$, and Fan ( 3$]$ ) has extended it in the framework of influential Kahane's T-martingale Theory. Fan (4]) also calculated the Hausdorff dimension of Riesz products. We are also motivated by the deterministic Riesz products on $\mathbb{Z}_{p}$, for which Fan and Zhang ([6]) have done a fairly complete study from the viewpoints of harmonic analysis and dynamical systems.

Now we state our main results as follows. Firstly, let $\sim$ and $\perp$ denote respectively the mutually absolute continuity and mutual singularity. To state Theorem 1.1, let

$$
s_{k}=\frac{1}{2 \pi} \arg \left(a_{k}-b_{k}\right), \quad t_{k}=\frac{1}{2 \pi} \arg \left(a_{k}+b_{k}\right), \quad u_{k}=\frac{a_{k}+b_{k}}{2},
$$

and $j_{k}$ be the unique index such that

$$
\left|\frac{j_{k}}{p^{k}}+t_{k}-\frac{1}{2}\right|=\min _{0 \leq j \leq p^{k}-1}\left|\frac{j}{p^{k}}+t_{k}-\frac{1}{2}\right|,
$$

in which the absolute value denotes the distance to the nearest integer. We note that the above value is $\leq \frac{1}{2 p^{k}}$. Now, set

$$
q_{k}=\frac{j_{k}}{p^{k}}+t_{k}-\frac{1}{2}
$$

Theorem 1.1. Suppose that $\left|a_{k}\right| \leq 1,\left|b_{k}\right| \leq 1$ for all $k \geq 1$. Then $\mu_{a, \omega} \sim \mu_{b, \omega}$ a.s. if

$$
\begin{aligned}
& \qquad \sum_{k=1}^{\infty}\left|a_{k}-b_{k}\right|^{2}\left(\frac{1}{1-\left|u_{k}\right| \cos \frac{\pi}{p^{k}}}+\frac{\cos ^{2} 2 \pi\left(s_{k}-t_{k}+q_{k}\right)}{p^{k}\left(1-\left|u_{k}\right| \cos 2 \pi q_{k}\right)}\right)<\infty ; \\
& \text { and } \mu_{a, \omega} \perp \mu_{b, \omega} \text { a.s. if } \\
& \qquad \sum_{k=1}^{\infty}\left|a_{k}-b_{k}\right|^{2}\left(1+\frac{\cos ^{2} 2 \pi\left(s_{k}-t_{k}+q_{k}\right)}{p^{k}\left(1-\left|u_{k}\right| \cos 2 \pi q_{k}\right)}\right)=\infty .
\end{aligned}
$$

Remark. Since both $\left|a_{k}\right| \leq 1$ and $\left|b_{k}\right| \leq 1$, we have $\left|u_{k}\right| \leq 1$ and $\left|u_{k}\right|=1$ only when $a_{k}=b_{k}$ with $\left|a_{k}\right|=1$, while in this case the corresponding summand in the above two series vanishes automatically.

Under an additional assumption that the decay rate of $\left(2-\left|a_{k}+b_{k}\right|\right)^{2}$ does not exceed $p^{-k}$, we have the following dichotomy. However, at this stage, we are not able to see whether this assumption is an optimal one to guarantee the dichotomy.

Theorem 1.2. Suppose that $\left|a_{k}\right| \leq 1,\left|b_{k}\right| \leq 1$ for all $k \geq 1$, and suppose that there exists some $c>0$ such that $p^{k}\left(2-\left|a_{k}+b_{k}\right|\right)^{2}>c$ for all large $k$ with $a_{k} \neq b_{k}$.

Then $\mu_{a, \omega} \sim \mu_{b, \omega}$ a.s. if

$$
\sum_{k=1}^{\infty}\left|a_{k}-b_{k}\right|^{2}\left(1+\frac{\cos ^{2} 2 \pi\left(s_{k}-t_{k}\right)}{\sqrt{2-\left|a_{k}+b_{k}\right|}}\right)<\infty,
$$

and $\mu_{a, \omega} \perp \mu_{b, \omega}$ a.s. otherwise.

Remark. The remark below Theorem 1.1 still applies.

Secondly, let $\operatorname{dim}_{\mathrm{H}} \mu$ denote the Hausdorff dimension of a measure on $\mathbb{Z}_{p}$ with respect to the $p$-adic norm $|\cdot|_{p}$. 
Theorem 1.3. We have, a.s.,

$$
\operatorname{dim}_{\mathrm{H}} \mu_{a, \omega}=1-\frac{1}{\log p} \cdot \limsup _{n \rightarrow \infty} \frac{1}{n} \sum_{k=1}^{n} \sigma_{k}\left(a_{k}\right)
$$

where

$$
\sigma_{k}\left(a_{k}\right)=p^{-k} \sum_{j=0}^{p^{k}-1}\left(1+\boldsymbol{R} \mathbf{e} a_{k} e^{2 \pi i \frac{j}{p^{k}}}\right) \log \left(1+\boldsymbol{R} \mathbf{e} a_{k} e^{2 \pi i \frac{j}{p^{k}}}\right) .
$$

Assume further that $a_{k}=a$ with $|a|<1$ for all $k \geq 1$. Then,

$$
\operatorname{dim}_{\mathrm{H}} \mu_{a, \omega}=1-\frac{\tau(a)}{\log p}, \quad \text { a.s., }
$$

where

$$
\tau(a)=\int_{0}^{1}\left(1+\mathbf{R e} a e^{2 \pi i x}\right) \log \left(1+\mathbf{R e} a e^{2 \pi i x}\right) d x,
$$

and in this case the convergence rate is dominated by

$$
\left|\sigma_{k}(a)-\sigma_{k-1}(a)\right| \leq \frac{C_{p, a}}{p^{k}}
$$

where $C_{p, a}$ is a constant.

Remark. In Theorem 1.3, we may relax the condition on the coefficients $a_{k}$ to assume both that $\sup \left|a_{k}\right|<1$ and that $\lim _{n \rightarrow \infty} \frac{1}{n} \sum_{k=1}^{n}\left|a_{k}-a\right|=0$, and we may still get the same integral representation on the Hausdorff dimension. However, we may then only get a poor convergence rate such as

$$
\left|\sigma_{k}\left(a_{k}\right)-\sigma_{k-1}\left(a_{k-1}\right)\right| \leq C_{p, a}\left(\frac{1}{p^{k}}+\left|a_{k}-a_{k-1}\right|\right) .
$$

To mention the significance, we remark that the conditions and the assertions in our theorems are specific to the $p$-adic case, which do not appear in previous literatures on the circle case, to our knowledge. Therefore, our theorems may compare significantly to the well-known circle case. We should remark that the work of Fan ([3]), which is based on T-martingale theory, is applied to any compact Abelian group, yet his general conditions may take different forms on different groups, and thus it is worthwhile to work out precise conditions for the particularly meaningful $p$-adic case (we thank A.H. Fan for bringing this to our attention).

In Section 2, we provide some preliminaries of random Riesz products and prove Theorems 1.1 and 1.2 via a proposition which may be of intrinsic interest. In Section 3, we provide some notions of Hausdorff dimension and prove Theorem 1.3.

A remark on $\mathbb{Z}_{p}$ : It is intriguing to ask whether the work in this paper should be carried out for a larger class of "adic" groups rather than merely for prime $p$ adic. Though we may modify most parts of the work to hold for the structure $\mathbb{Z}_{n}$ of general positive integer $n \geq 2$, we are leaning towards regarding the Riesz product as an object defined for a "simple" group $\mathbb{Z}_{p}$ (inherited from the local field $\mathbb{Q}_{p}$ ). In the case of, say, $\mathbb{Z}_{6}$, we may instead develop our results on the product group $\mathbb{Z}_{2} \oplus \mathbb{Z}_{3}$, which could be potentially interesting, and some deterministic theory is also sketched in the last section of Peyrière [13. We thank the referee for bringing this observation to our attention. 


\section{Preliminaries, mutually absolute continuity AND MUTUAL SINGULARITY}

We use the notation in Section 1 and define, for each $n=1,2,3, \cdots$,

$$
Q_{a, n}(x)=Q_{a, n}(x, \omega)=\prod_{k=1}^{n}\left(1+\boldsymbol{R e} a_{k} \gamma_{k}\left(x+\omega_{k}\right)\right)
$$

where $a:=\left\{a_{k}\right\}, k \geq 1$, is a sequence of complex numbers with $\left|a_{k}\right| \leq 1$ and $\gamma_{k}$ is a sequence of characters of the additive group $\mathbb{Z}_{p}$ which we take, in this paper, to be $\gamma_{k}(t)=\exp \left(2 \pi i\left\{p^{-k} t\right\}\right)$. We remark that $\left\{\gamma_{k}: k \geq 1\right\}$ is only a subset of the dual group $\widehat{\mathbb{Z}}_{p}$; see [15, 16]. We recall that each number $x \in \mathbb{Q}_{p}$ has a unique expansion in the following form:

$$
x=\sum_{j=-m}^{\infty} x_{j} p^{j}, x_{j} \in\{0,1, \cdots, p-1\}, m \in \mathbb{Z} .
$$

Then, we use $\{x\}$ to denote the rational number $\sum_{j=-m}^{-1} x_{j} p^{j}$ associated with $x$, and we define that $|x|_{p}:=p^{n}$ whenever $x_{-n} \neq 0$.

We denote by $d x$ the normalized Haar measure on $\mathbb{Z}_{p}$. By the Kahane's T-martingale theory ([7]), it is known that the random probability measures $Q_{a, n}(x) d x$, for a.s. $\omega$, converge weakly to a non-degenerate random measure on $\mathbb{Z}_{p}$, denoted by $\mu_{a, \omega}$, which is called a random p-adic Riesz product (measure). We write formally

$$
d \mu_{a, \omega}=\prod_{k=1}^{\infty}\left(1+\boldsymbol{R e} a_{k} \gamma_{k}\left(x+\omega_{k}\right)\right) d x
$$

The measure $\mu_{a, \omega}$ is, for almost sure $\omega$, a measure of total mass one. This can be obtained by the T-martingale theory (see, for example, [3]) or by the dissociation of characters. The essential methods and results in this paper are probabilistic, though we believe that the analytic approach could be used to obtain the parallel results by the methodology of the dissociation of characters (we thank the referee for pointing out the dissociation to us).

Let $\mu_{a, \omega}$ and $\mu_{b, \omega}$ be the random $p$-adic Riesz products defined by coefficients $a:=\left\{a_{k}\right\}$ and $b:=\left\{b_{k}\right\}$ respectively. In this section, we will discuss mutual absolute continuity and mutual singularity of $\mu_{a, \omega}$ and $\mu_{b, \omega}$, which will lead to the proofs of Theorems 1.1 and 1.2.

Notice: In the following context, we only concern ourselves with those $k$ 's for which $a_{k} \neq b_{k}$ (see the remark below Theorem 1.1 in Section 1) and thus all $u_{k}:=\left(a_{k}+b_{k}\right) / 2$ are of $\left|u_{k}\right|<1$ in the following context.

We state a dichotomy criterion for random Riesz products as follows, in which there is no decay assumption imposed on $a$ and $b$. The proposition can be traced back to the seminal [8]. Let

$$
L_{k}\left(a_{k}, b_{k}\right)=\frac{1}{p^{k}} \sum_{j=0}^{p^{k}-1} \frac{\cos ^{2} 2 \pi\left(\frac{j}{p^{k}}+s_{k}\right)}{1+\left|u_{k}\right| \cos 2 \pi\left(\frac{j}{p^{k}}+t_{k}\right)},
$$

where $s_{k}, t_{k}, u_{k}$ are those quantities defined in Section 1. We have 
Proposition 2.1. Suppose that $\left|a_{k}\right| \leq 1,\left|b_{k}\right| \leq 1$ for all $k \geq 1$. Then $\mu_{a, \omega} \sim \mu_{b, \omega}$ a.s. if

$$
\sum_{k=1}^{\infty} L_{k}\left(a_{k}, b_{k}\right)\left|a_{k}-b_{k}\right|^{2}<\infty
$$

and $\mu_{a, \omega} \perp \mu_{b, \omega}$ a.s. otherwise.

Proof. Write

$$
I_{k}\left(a_{k}, b_{k}\right)=\int_{\mathbb{Z}_{p}} \sqrt{\left(1+\boldsymbol{R} \mathbf{e} a_{k} \gamma_{k}(x)\right)\left(1+\boldsymbol{\operatorname { R e }} b_{k} \gamma_{k}(x)\right)} d x .
$$

By Theorem 2.1 in Fan 3 , (which is adapted from those in [8, 9]), stochastic independence of the $\omega_{k}$ 's and translation-invariance of the Haar measure, we have

$$
\begin{aligned}
& \mu_{a, \omega} \sim \mu_{b, \omega} \quad \text { a.s. } \Longleftrightarrow \prod_{k=1}^{\infty} I_{k}\left(a_{k}, b_{k}\right)>0, \\
& \mu_{a, \omega} \perp \mu_{b, \omega} \quad \text { a.s. } \Longleftrightarrow \prod_{k=1}^{\infty} I_{k}\left(a_{k}, b_{k}\right)=0 .
\end{aligned}
$$

To estimate the above products, we note that $\mathbb{Z}_{p}$ can be expressed as a collection of $p^{k}$ disjoint balls with the same size. Namely,

$$
\mathbb{Z}_{p}=\bigsqcup_{j=0}^{p^{k}-1} B_{k}(j)
$$

Moreover, $\gamma_{k}(x)$ takes a constant $e^{2 \pi i \frac{j}{p^{k}}}$ on each ball $B_{k}(j)$. Hence

$$
I_{k}\left(a_{k}, b_{k}\right)=\frac{1}{p^{k}} \sum_{j=0}^{p^{k}-1} \sqrt{\left(1+\boldsymbol{\operatorname { R e }} a_{k} e^{2 \pi i \frac{j}{p^{k}}}\right)\left(1+\boldsymbol{\operatorname { R e }} b_{k} e^{2 \pi i \frac{j}{p^{k}}}\right)} .
$$

Denote by $I_{k}\left(a_{k}, b_{k}, j\right)$ the general term in the last sum. Write

$$
\begin{aligned}
I_{k}\left(a_{k}, b_{k}, j\right) & =\sqrt{\left(1+\xi_{k, j}+\eta_{k, j}\right)\left(1+\xi_{k, j}-\eta_{k, j}\right)} \\
& =\left(1+\xi_{k, j}\right) \sqrt{1-\left(\frac{\eta_{k, j}}{1+\xi_{k, j}}\right)^{2}},
\end{aligned}
$$

where

$$
\xi_{k, j}=\mathbf{R e} \frac{a_{k}+b_{k}}{2} e^{2 \pi i \frac{j}{p^{k}}}, \quad \eta_{k, j}=\mathbf{R e} \frac{a_{k}-b_{k}}{2} e^{2 \pi i \frac{j}{p^{k}}} .
$$

Let $s_{k}$ and $t_{k}$ be the two argument numbers associated with $a$ and $b$, as defined in Section 1 , and

$$
L_{k}\left(a_{k}, b_{k}\right)=\frac{1}{p^{k}} \sum_{j=0}^{p^{k}-1} \frac{\cos ^{2} 2 \pi\left(\frac{j}{p^{k}}+s_{k}\right)}{1+\left|\left(a_{k}+b_{k}\right) / 2\right| \cos 2 \pi\left(\frac{j}{p^{k}}+t_{k}\right)} .
$$

Now, using the inequalities $1-x \leq \sqrt{1-x} \leq 1-\frac{x}{2}$ for all $x \in[0,1]$ and the fact that $\sum_{j=0}^{p^{k}-1} \xi_{k, j}=0$, we get

$$
1-\frac{1}{4}\left|a_{k}-b_{k}\right|^{2} L_{k}\left(a_{k}, b_{k}\right) \leq I_{k}\left(a_{k}, b_{k}\right) \leq 1-\frac{1}{8}\left|a_{k}-b_{k}\right|^{2} L_{k}\left(a_{k}, b_{k}\right) .
$$


Hence by (2.1) and the left side of (2.3), we obtain the first assertion on the mutual absolute continuity (note that $I_{k}$ is symmetric in $a_{k}, b_{k}$ ). At the same time, the mutual singularity is a direct consequence of (2.2) and the right side of (2.3) . This completes the proof of Proposition 2.1.

The apparent drawback of Proposition 2.1 is that $L_{k}\left(a_{k}, b_{k}\right)$ is not readily expressed. We proceed with some proper reshaping, which will lead to the proofs of Theorems 1.1 and 1.2.

Proof of Theorem 1.1. We divide $L_{k}$ into two parts. Let

$$
L_{k}^{\prime}=\frac{1}{p^{k}} \sum_{j \neq j_{k}} \frac{\cos ^{2} 2 \pi\left(\frac{j}{p^{k}}+s_{k}\right)}{1+\left|u_{k}\right| \cos 2 \pi\left(\frac{j}{p^{k}}+t_{k}\right)}, \quad L_{k}^{\prime \prime}=\frac{1}{p^{k}} \cdot \frac{\cos ^{2} 2 \pi\left(\frac{j_{k}}{p^{k}}+s_{k}\right)}{1+\left|u_{k}\right| \cos 2 \pi\left(\frac{j_{k}}{p^{k}}+t_{k}\right)} .
$$

Hence

$$
\begin{aligned}
L_{k}^{\prime} & \geq \frac{1}{p^{k}} \sum_{j \neq j_{k}} \frac{\cos ^{2} 2 \pi\left(\frac{j}{p^{k}}+s_{k}\right)}{2} \geq \frac{1}{p^{k}}\left(\sum_{j=0}^{p^{k}-1} \frac{\cos ^{2} 2 \pi\left(\frac{j}{p^{k}}+s_{k}\right)}{2}-\frac{1}{2}\right) \\
& =\frac{1}{p^{k}}\left(\frac{p^{k}}{4}-\frac{1}{2}\right) \geq \frac{1}{8}
\end{aligned}
$$

for all $k \geq 2, p \geq 2$. On the other hand,

$$
\begin{aligned}
L_{k}^{\prime} & \leq \frac{1}{p^{k}} \sum_{j \neq j_{k}} \frac{\cos ^{2} 2 \pi\left(\frac{j}{p^{k}}+s_{k}\right)}{1-\left|u_{k}\right| \cos \frac{\pi}{p^{k}}} \leq \frac{1}{p^{k}} \sum_{j=0}^{p^{k}-1} \frac{\cos ^{2} 2 \pi\left(\frac{j}{p^{k}}+s_{k}\right)}{1-\left|u_{k}\right| \cos \frac{\pi}{p^{k}}} \\
& =\frac{1}{2\left(1-\left|u_{k}\right| \cos \frac{\pi}{p^{k}}\right)} .
\end{aligned}
$$

Now we consider $L_{k}^{\prime \prime}$ and recall that $q_{k}=\frac{j_{k}}{p^{k}}+t_{k}-\frac{1}{2}$. Then

$$
L_{k}^{\prime \prime}=\frac{1}{p^{k}} \cdot \frac{\cos ^{2} 2 \pi\left(s_{k}-t_{k}+q_{k}\right)}{1-\left|u_{k}\right| \cos 2 \pi q_{k}} .
$$

Hence the assertions of Theorem 1.1 are a consequence of (2.4), (2.5), (2.6) and Proposition 2.1.

Proof of Theorem 1.2. We observe that $L_{k}\left(a_{k}, b_{k}\right)$ can be rewritten as

$$
L_{k}\left(a_{k}, b_{k}\right)=\sum_{j=0}^{p^{k}-1} \int_{\frac{j}{p^{k}}}^{\frac{j+1}{p^{k}}} \frac{\cos ^{2} 2 \pi\left(\frac{j}{p^{k}}+s_{k}\right)}{1+\left|u_{k}\right| \cos 2 \pi\left(\frac{j}{p^{k}}+t_{k}\right)} d x
$$

Let

$$
H_{k}\left(a_{k}, b_{k}\right)=\int_{0}^{1} \frac{\cos ^{2} 2 \pi\left(x+s_{k}\right)}{1+\left|u_{k}\right| \cos 2 \pi\left(x+t_{k}\right)} d x=\sum_{j=0}^{p^{k}-1} \int_{\frac{j}{p^{k}}}^{\frac{j+1}{p^{k}}} \frac{\cos ^{2} 2 \pi\left(x+s_{k}\right)}{1+\left|u_{k}\right| \cos 2 \pi\left(x+t_{k}\right)} d x .
$$

It is easy to obtain that

$$
\left|\frac{\cos ^{2} 2 \pi\left(\frac{j}{p^{k}}+s_{k}\right)}{1+\left|u_{k}\right| \cos 2 \pi\left(\frac{j}{p^{k}}+t_{k}\right)}-\frac{\cos ^{2} 2 \pi\left(x+s_{k}\right)}{1+\left|u_{k}\right| \cos 2 \pi\left(x+t_{k}\right)}\right| \leq \frac{C}{p^{k}\left(1-\left|u_{k}\right|\right)^{2}}
$$


for all $x \in\left[\frac{j}{p^{k}}, \frac{j+1}{p^{k}}\right]$, where $C$ is an absolute constant. Hence, under our additional assumption on $a_{k}, b_{k}$, we have $\left|L_{k}\left(a_{k}, b_{k}\right)-H_{k}\left(a_{k}, b_{k}\right)\right| \leq 2 C / c$, whence

$$
\sum_{k=1}^{\infty} L_{k}\left(a_{k}, b_{k}\right)\left|a_{k}-b_{k}\right|^{2}<\infty \Longleftrightarrow \sum_{k=1}^{\infty} H_{k}\left(a_{k}, b_{k}\right)\left|a_{k}-b_{k}\right|^{2}<\infty,
$$

since, if $\sum\left|a_{k}-b_{k}\right|^{2}=\infty$, then both series diverge. We then apply a direct formula for $H_{k}\left(a_{k}, b_{k}\right)$ (see [9]):

$$
H_{k}\left(a_{k}, b_{k}\right)=\frac{\cos ^{2} 2 \pi\left(s_{k}-t_{k}\right)}{\sqrt{1-\left|u_{k}\right|^{2}}+1-\left|u_{k}\right|^{2}}+\frac{\sin ^{2} 2 \pi\left(s_{k}-t_{k}\right)}{\sqrt{1-\left|u_{k}\right|^{2}}+1} .
$$

We observe that

$$
H_{k}\left(a_{k}, b_{k}\right) \leq \frac{\cos ^{2} 2 \pi\left(s_{k}-t_{k}\right)}{\sqrt{1-\left|u_{k}\right|}}+1 \leq 4 \sqrt{2} H_{k}\left(a_{k}, b_{k}\right) .
$$

Therefore the assertions of Theorem 1.2 follow from the corresponding results in $H_{k}\left(a_{k}, b_{k}\right)$.

\section{HAUSDORFF DIMENSION}

Recall that, since $\mathbb{Z}_{p}$ is equipped with the $p$-adic norm $|\cdot|_{p}$, the Hausdorff dimension of a set is well defined (see [2, 11]). The Hausdorff dimension of $\mu_{a, \omega}$, denoted by $\operatorname{dim}_{\mathrm{H}} \mu_{a, \omega}$, is defined to be the infimum of the $\operatorname{dim} E$ 's such that $\mu_{a, \omega}(E)=1$ (see [5, 11, for more details).

Before calculating the Hausdorff dimension of $\mu_{a, \omega}$, we need to introduce a new measure called the Peyrière measure ([7]), which may also be regarded as a kind of Palm distribution. This is the unique probability measure $\mathbf{q}$ on the $\sigma$-field generated by the $B \times A\left(B\right.$ : Borel set in $\mathbb{Z}_{p}, A$ : event in $\left.\Omega\right)$ which satisfies

$$
\mathbb{E}_{\mathbf{q}} f:=\int_{\mathbb{Z}_{p} \times \Omega} f(x, \omega) d \mathbf{q}(x, \omega)=\mathbb{E}_{\mathbf{P}} \int_{\mathbb{Z}_{p}} f(x, \omega) d \mu_{a, \omega}
$$

for all positive measurable functions $f(x, \omega)$. Then we have the following lemma which is a consequence of the Kolmogorov Three-Series Theorem (see Theorem 1.5 in [4]).

Lemma 3.1. Suppose that $f_{k}, k \geq 1$, is a sequence of functions in $L^{2}(d x)$. Then almost surely the series

$$
\sum_{k=1}^{\infty}\left(f_{k}\left(x+\omega_{k}\right)-\mathbb{E}_{\mathbf{q}} f_{k}\left(x+\omega_{k}\right)\right)
$$

converges for $\mu_{a, \omega}$-a.e. $x$ if and only if the series

$$
\sum_{k=1}^{\infty}\left(M_{k}-m_{k}^{2}\right)
$$

converges, where

$$
M_{k}=\int_{\mathbb{Z}_{p}} f_{k}^{2}(x)\left(1+\boldsymbol{\operatorname { R e }} a_{k} \gamma_{k}(x)\right) d x ; \quad m_{k}=\int_{\mathbb{Z}_{p}} f_{k}(x)\left(1+\boldsymbol{\operatorname { R e }} a_{k} \gamma_{k}(x)\right) d x .
$$


Now we apply Lemma 3.1 to calculate the Hausdorff dimension of $\mu_{a, \omega}$. Recall that the Hausdorff dimension of a measure $\mu$ is defined by $\operatorname{dim}_{\mathrm{H}} \mu=\inf \left\{\operatorname{dim}_{\mathrm{H}} E\right.$ : $E$ a Borel set and $\left.\mu\left(E^{c}\right)=0\right\}$ (see [5]). Let $B_{n}(x)=\left\{y \in \mathbb{Z}_{p}:|x-y|_{p} \leq p^{-n}\right\}$. Then the dimension $\operatorname{dim}_{\mathrm{H}} \mu$ is equal to the essential supremum of the lower local density

$$
\underline{D}(\mu, x)=\liminf _{n \rightarrow \infty} \frac{\log \mu\left(B_{n}(x)\right)}{\log \left|B_{n}(x)\right|},
$$

where $\left|B_{n}(x)\right|$ denotes the Haar measure of $B_{n}(x)$ (see [5]).

From the definition of $\mu_{a, \omega}$, for a.s. $\omega$, for any given $n$ and $x$,

$$
\mu_{a, \omega}\left(B_{n}(x)\right)=\lim _{N \rightarrow \infty} \int_{B_{n}(x)} Q_{a, N}(y) d y=p^{-n} Q_{a, n}(x) .
$$

The reason for the equality in the above display is that $y \mapsto Q_{a, N}(x+y) / Q_{a, n}(x)$ is a finite Riesz product on the subgroup $B_{n}(0)$, and thus its integral is equal to 1 for the normalized Haar measure and to $p^{-n}$ for the induced measure (here, we thank the referee for pointing out an error and providing the correct argument for this equality).

Hence

$$
\underline{D}\left(\mu_{a, \omega}, x\right)=1-\frac{1}{\log p} \cdot \limsup _{n \rightarrow \infty} \frac{1}{n} \sum_{k=1}^{n} \log \left(1+\boldsymbol{R} \mathbf{e} a_{k} \gamma_{k}\left(x+\omega_{k}\right)\right) .
$$

Now take

$$
f_{k}(x)=\frac{1}{k} \log \left(1+\boldsymbol{R e} a_{k} \gamma_{k}(x)\right)
$$

in Lemma 3.1 and let

$$
\sigma_{k}\left(a_{k}\right)=\int_{\mathbb{Z}_{p}}\left(1+\boldsymbol{R e} a_{k} \gamma_{k}(x)\right) \log \left(1+\boldsymbol{R e} a_{k} \gamma_{k}(x)\right) d x .
$$

Then $\mathbb{E}_{\mathbf{q}} f_{k}\left(x+\omega_{k}\right)=\frac{1}{k} \sigma_{k}\left(a_{k}\right)$. By Lemma 3.1 and Kronecker's lemma, almost surely

$$
\frac{1}{n} \sum_{k=1}^{n}\left[\log \left(1+\boldsymbol{R} \mathbf{e} a_{k} \gamma_{k}\left(x+\omega_{k}\right)\right)-\sigma_{k}\left(a_{k}\right)\right]
$$

converges to zero for $\mu_{a, \omega}$-a.e. $x$. Thus, almost surely

$$
\underline{D}\left(\mu_{a, \omega}, x\right)=1-\frac{1}{\log p} \cdot \limsup _{n \rightarrow \infty} \frac{1}{n} \sum_{k=1}^{n} \sigma_{k}\left(a_{k}\right), \quad \mu_{a, \omega} \text {-a.e. } \quad x .
$$

By the definition of Hausdorff dimension of a measure, we have, a.s.,

$$
\operatorname{dim}_{\mathrm{H}} \mu_{a, \omega}=1-\frac{1}{\log p} \cdot \limsup _{n \rightarrow \infty} \frac{1}{n} \sum_{k=1}^{n} \sigma_{k}\left(a_{k}\right) .
$$

Now we explore the integral $\sigma_{k}\left(a_{k}\right)$. By the same argument as has been done for $I_{k}\left(a_{k}, b_{k}\right)$, we obtain

$$
\sigma_{k}\left(a_{k}\right)=p^{-k} \sum_{j=0}^{p^{k}-1}\left(1+\boldsymbol{\operatorname { R e }} a_{k} e^{2 \pi i \frac{j}{p^{k}}}\right) \log \left(1+\boldsymbol{R} \mathbf{e} a_{k} e^{2 \pi i \frac{j}{p^{k}}}\right) .
$$


Under the assumption that $a_{k}=a$ with $|a|<1$ for all $k \geq 1$, the above is a Riemann sum, and thus

$$
\lim _{k \rightarrow \infty} \sigma_{k}(a)=\int_{0}^{1}\left(1+\boldsymbol{R} \mathbf{e} a e^{2 \pi i x}\right) \log \left(1+\mathbf{R e} a e^{2 \pi i x}\right) d x:=\tau(a) .
$$

It follows that, almost surely,

$$
\operatorname{dim}_{\mathrm{H}} \mu_{a, \omega}=1-\frac{\tau(a)}{\log p} .
$$

As for the convergence rate in this case, we calculate that

$$
\left|\sigma_{k}(a)-\sigma_{k-1}(a)\right| \leq \frac{C_{p, a}}{p^{k}},
$$

where $C_{p, a}$ is a constant specified below, as follows. We identify $\mathbb{Z} / p \mathbb{Z}$ as $\{0, \cdots$, $p-1\}$ and the integration over $\mathbb{Z} / p \mathbb{Z}$ as the summation over $j \in\{0, \cdots, p-1\}$ divided by $p$. Then we rewrite $\sigma_{k}(a)$ formally as a multiple integral:

$$
\begin{aligned}
& \sigma_{k}(a)= \int_{(\mathbb{Z} / p \mathbb{Z})^{k}}\left(1+\operatorname{Re} a e^{2 \pi i \frac{x_{0}+x_{1} p+\cdots+x_{k-1} p^{k-1}}{p^{k}}}\right) \\
& \cdot \log \left(1+\boldsymbol{R e} a e^{2 \pi i \frac{x_{0}+x_{1} p+\cdots+x_{k-1} p^{k-1}}{p^{k}}}\right) d x_{0} d x_{1} \cdots d x_{k-1} \\
&=\int_{\mathbb{Z} / p \mathbb{Z}} d x_{0} \int_{(\mathbb{Z} / p \mathbb{Z})^{k-1}}\left(1+\operatorname{Re} a e^{2 \pi i \frac{x_{0}}{p^{k}}} e^{2 \pi i \frac{x_{1}+x_{2} p+\cdots+x_{k-1} p^{k-2}}{p^{k-1}}}\right) \\
& \cdot \log \left(1+\operatorname{Re} a e^{2 \pi i \frac{x_{0}}{p^{k}}} e^{2 \pi i \frac{x_{1}+x_{2} p+\cdots+x_{k-1} p^{k-2}}{p^{k-1}}}\right) d x_{1} d x_{2} \cdots d x_{k-1} .
\end{aligned}
$$

Using the above formal integration, we may proceed with a certain change of variables to obtain that

$$
\sigma_{k}(a)=\int_{\mathbb{Z} / p \mathbb{Z}} d t \int_{\mathbb{Z}_{p}}\left(1+\boldsymbol{R} \mathbf{e} a e^{2 \pi i \frac{t}{p^{k}}} \gamma_{k-1}(x)\right) \log \left(1+\boldsymbol{R} \mathbf{e} a e^{2 \pi i \frac{t}{p^{k}}} \gamma_{k-1}(x)\right) d x .
$$

Note that $\sigma_{k-1}(a)$ can also be written in the integral form as

$$
\sigma_{k-1}(a)=\int_{\mathbb{Z} / p \mathbb{Z}} d t \int_{\mathbb{Z}_{p}}\left(1+\boldsymbol{R} \mathbf{e} a \gamma_{k-1}(x)\right) \log \left(1+\boldsymbol{R} \mathbf{e} a \gamma_{k-1}(x)\right) d x .
$$

We observe that the absolute value of the difference of the two integrands in the above two integral displays is uniformly dominated by $p^{-k}(p-1)(2-\log (1-|a|))$. Therefore, the assertion on the convergence rate in Theorem 1.3 follows from the above estimate.

\section{ACKNOWLEDGEMENTS}

This work was finished while the second author did his postdoctoral research at National Taiwan University, supported by grant NSC 97-2811-M-002-005. The first author is supported in part by grant NSC 96-2115-M-002-005-MY3, and the second author is supported in part by Mathematical Tian-Yuan Fund No. 10826054. We are grateful to the referee for a careful reading and constructive comments, which improved the precision of this paper. 


\section{REFERENCES}

[1] Brown, G. and Moran, W. (1974). On orthogonality of Riesz products. Proc. Camb. Phil. Soc. 76, 173-181. MR0350319 (50:2812)

[2] Falconer, K. (2003). Fractal geometry. Mathematical foundations and applications. Second edition. John Wiley \& Sons, Inc., Hoboken, NJ. MR2118797(2006b:28001)

[3] Fan, A. H. (1991). Équivalence et orthogonalité des mesures aléatoires engendrées par martingales positives homogènes. Studia Math. 98, 249-266. MR.1115195 (93d:60082)

[4] Fan, A. H. (1993). Quelques propriétés des produits de Riesz. Bull. Sci. Math. 117, 421-439. MR.1245805 (95f:28002)

[5] Fan, A. H. (1994). Sur les dimensions de mesures. Studia Math. 111, 1-17. MR.1292850 (95m:28003)

[6] Fan, A. H. and Zhang, X. Y. (2009) Some properties of Riesz products on the ring of $p$-adic integers. J. Fourier Anal. Appl. (to appear).

[7] Kahane, J. P. (1987). Positive martingales and random measures. Chin. Ann. Math. 8B (1), 1-12. MR886744 (88j:60098)

[8] Kakutani, S. (1948). On equivalence of infinite product measures. Ann. of Math. (2) 49, 214-224. MR0023331 (9:340e)

[9] Kilmer, S. J. and Saeki, S. (1988). On Riesz product measures: Mutual absolute continuity and singularity. Ann. Inst. Fourier (Grenoble) 38, 63-93. MR949011 (90a:42006)

[10] Koblitz, N. (1984). p-adic numbers, $p$-adic analysis, and zeta functions. Grad. Texts in Math., 58. Springer-Verlag. MR754003 (86c:11086)

[11] Mattila, P. (1995). Geometry of sets and measures in Euclidean spaces: Fractals and rectifiability. Cambridge Studies in Advanced Mathematics, 44. Cambridge University Press. MR1333890 (96h:28006)

[12] Parreau, F. (1990). Ergodicité et pureté des produits de Riesz. Ann. Inst. Fourier (Grenoble) 40, 391-405. MR1070833 (91g:42009)

[13] Peyrière, J. (1975). Étude de quelques propriétés des produits de Riesz. Ann. Inst. Fourier (Grenoble) 25, 127-169. MR0404973 (53:8771)

[14] Schikhof, W. H. (1984). Ultrametric calculus. Cambridge University Press. MR.791759 $(86 \mathrm{j}: 11104)$

[15] Taibleson, M. H. (1975). Fourier analysis on local fields. Mathematical Notes, Princeton University Press. MR0487295 (58:6943)

[16] Vladimirov, V. S., Volovich, I. V. and Zelenov, E. I. (1994). p-adic analysis and mathematical physics. World Scientific. MR.1288093 (95k:11155)

Department of Mathematics, National Taiwan University, Taipei 10617, Taiwan

E-mail address: shiehnr@math.ntu.edu.tw

Department of Mathematics, South China University of Technology, 510640 Guangzhou, People's Republic of China

E-mail address: xiongyzh@scut.edu.cn 